\title{
Development of a Drug Smoke Sensing System
}

\author{
Cheng-Chun Wu, Shih-Wen Chiu, Hsiang-Chiu Wu, Kea-Tiong Tang \\ Department of Electrical Engineering, National Tsing Hua University, Taiwan, \\ kttang@ee.nthu.edu.tw
}

\begin{abstract}
:
The commonest method of taking ketamine is to smoke cigarettes containing ketamine. Ketamine endangers health and dwelling quality in Taiwan, but there is no convenient method to detect ketamine immediately. We developed a gas sensing system to detect ketamine, and the experiment results indicated that the system could identify the gas of ketamine, cigarettes, and ketamine cigarettes.
\end{abstract}

Key words: ketamine, cigarette, gas sensing system, drug, smoke

\section{Introduction}

Smoking cigarettes containing ketamine vaporizes an odor smelling like burning plastic. This severely endangers health and dwelling quality in Taiwan. Ketamine is classified as a Class 3 drug. Police has no objective basis for obtaining search warrants to search houses from which the odor of ketamine emanates. To prevent this situation and enable the police or public to adduce evidence, developing effective and convenient methods for immediately detecting and identifying ketamine smoke is necessary.

Gas sensing has been used in many applications [1]. Usually, we use gas chromatography-mass spectrometry or Fourier transform-infrared spectroscopy to detecting and identifying drugs, but these instruments are expensive and not portable. Therefore, we developed a gas sensing system to detect the smoke of ketamine and ketamine cigarette.

\section{Ketamine Experiment Setup}

The gas sensing array consisted of 7 metal oxide sensors: the TGS-2600, TGS-2602, TGS2603, TGS-2610, TGS-2611, TGS-2612, and TGS-2620. The experiment process was as follows:

(1) Ketamine or ketamine cigarette was placed into a flask.
(2) The flask was heated to vaporize ketamine completely.

(3) Sampling gas and air was extracted using pumps in two paths separately.

(4) The flow rate was maintained at $300 \mathrm{cc} / \mathrm{min}$.

(5) After $3 \mathrm{~min}$, the sensor array output was recorded.

(6) The sensor array's resistance variation rate $\Delta \mathrm{Rs} / \mathrm{Rs} 0$ was normalized.

Figure 1 depicts the experiment setup.

\section{Experiment Results}

The test gases were divided into three classes: ketamine, cigarette, and ketamine cigarette.

Figure 2 shows the resulting patterns for the testing gases. The seven vertices of a heptagon were the average normalized resistance variation rate of seven metal oxide sensors. The resulting patterns for the three testing gases are totally different.

Also, Figure 3 shows the normalized sensor resistance variation rate of ketamine in different weights. There is a linear relationship between the resistance variation and ketamine in different weights.

The system could identify the gas of ketamine gas, cigarettes, and ketamine cigarettes. 


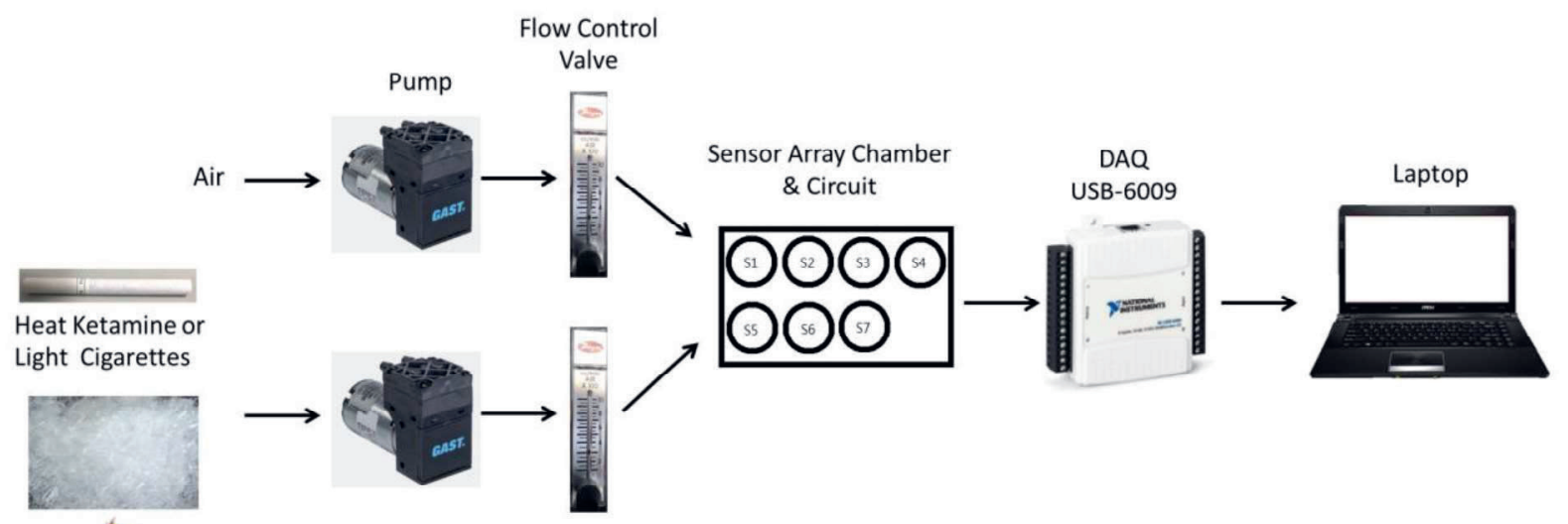

Fig.1. The experiment setup.

(a)

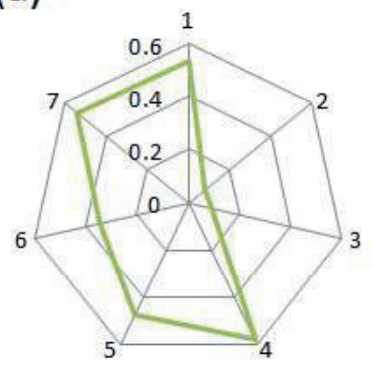

(b)

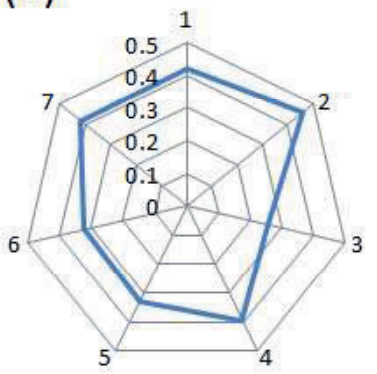

(c)

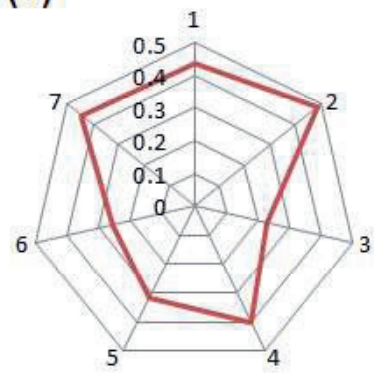

Fig.2. The resulting patterns for the testing gases. (a) ketamine; (b) cigarette; (c) ketamine cigarette

$$
(\Delta \mathrm{Rs}) / \mathrm{Rs} 0
$$

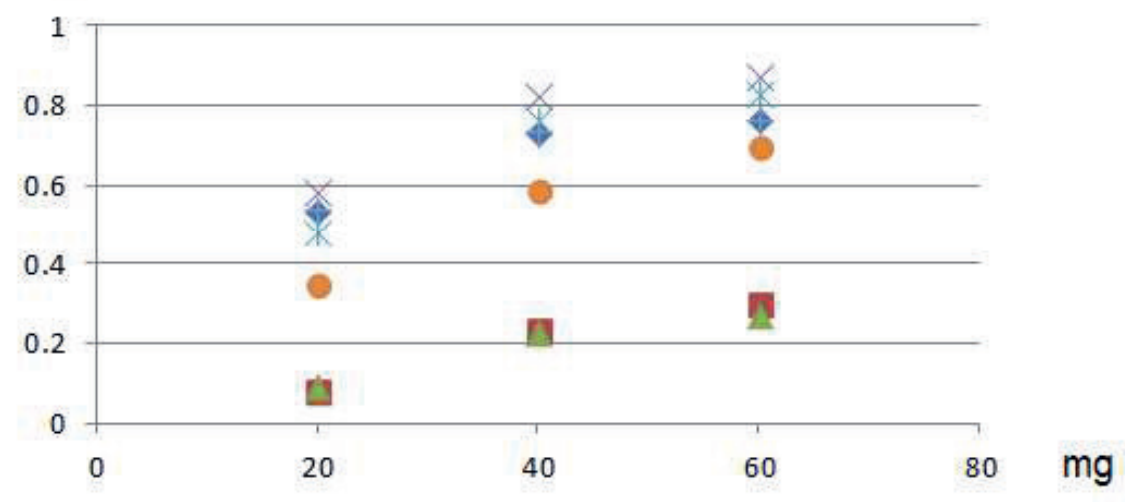

Fig.3. The normalized sensor resistance variation rate of ketamine of different weights.

\section{Acknowledgments}

This work was supported in part by the Ministry of Science and Technology, under Contract No. MOST 106-2221-E-007-119. The authors would like to thank Professor Sheng-Meng Wang for providing ketamine samples. (Department of Forensic Science, Central Police University, Taiwan)

\section{References}

[1] Harwood, D., Something in the air [electronic nose]. IEE Review 2001, 47, (1), 10-14. 